\title{
Erster SAQM Qualitätsbericht 2017 veröffentlicht!
}

\section{Roxane Kübler ${ }^{a}$, Esther Kraft ${ }^{b}$, Christoph Bosshard ${ }^{c}$}

${ }^{a}$ MA, Operative Leiterin der Schweizerischen Akademie für Qualität in der Medizin SAQM der FMH; ${ }^{b}$ lic. rer. oec., Leiterin Abteilung Daten, Demographie und Qualität DDQ der FMH; ${ }^{\circ}$ Dr. med., Vizepräsident der FMH, Departementsverantwortlicher Daten, Demographie und Qualität

Der erste Qualitätsbericht der Schweizerischen Akademie für Qualität in der Medizin SAQM ist nun veröffentlicht. Der Bericht weist aus, wie vielfältig die Qualitätsarbeit der Ärzteschaft ist, und schafft Transparenz gegen innen und aussen und damit auch die Möglichkeit, dass sich Organisationen untereinander vernetzen und Synergien nutzen. Für die FMH ist zentral, dass auch bei der Qualitätsarbeit auf Bestehendem aufgebaut und das vorhandene Wissen sinnvoll genutzt wird. Damit dies nachhaltig gewährleistet werden kann, ist mitunter eine gesicherte Finanzierung - über das KVG hinaus - unabdingbar. Mit der Unterzeichnung der QualitätsCharta SAQM verpflichten sich aktuell 69 Ärzteorganisationen zu Transparenz, Verbindlichkeit und Nachhaltigkeit. Dazu gehört auch die Erstellung eines Qualitätsberichts und einer Qualitätsstrategie. 2017 veröffentlichte die SAQM die eigene Qualitätsstrategie. Darauf aufbauend erarbeitete sie den vorliegenden SAQM Qualitätsbericht für das Jahr 2017.

\section{Schweizerische Akademie für Qualität in der Medizin SAQM}

Sie haben sich entschieden, dem Qualitätsbericht der SAQM, der Qualitätsorganisation der FMH, Ihre Aufmerksamkeit zuzuwenden. Dafür danken wir Ihnen und sind überzeugt, dass Sie beim Lesen auf viel Interessantes stossen werden, welches in diesem Bericht lediglich schlaglichtartig beleuchtet werden kann. Wenn es uns gelingt, am einen oder anderen Ort Ihr Interesse für zusätzliche Informationen zu wecken, so führen Sie die jeweiligen Links weiter. Gerne stehen wir Ihnen auch für Ihre Fragen und Anliegen zur Verfügung!

2012 gründete die FMH die Schweizerische Akademie für Qualität in der Medizin SAQM. Als die Qualitätsorganisation der Schweizer Ärzteschaft ist sie zuständig für alle Belange der Qualität in der Medizin. Sie hat zum Ziel, Qualitätsaktivitäten zu vernetzen und zu koordinieren und damit Doppelspurigkeiten zu vermeiden sowie Synergien zu nutzen.

Mit dem vorliegenden Qualitätsbericht weist die SAQM aus, wie vielfältig die Qualitätsarbeit der Ärzteschaft ist. Die Kurzversion ist in Form dieses Artikels publi-

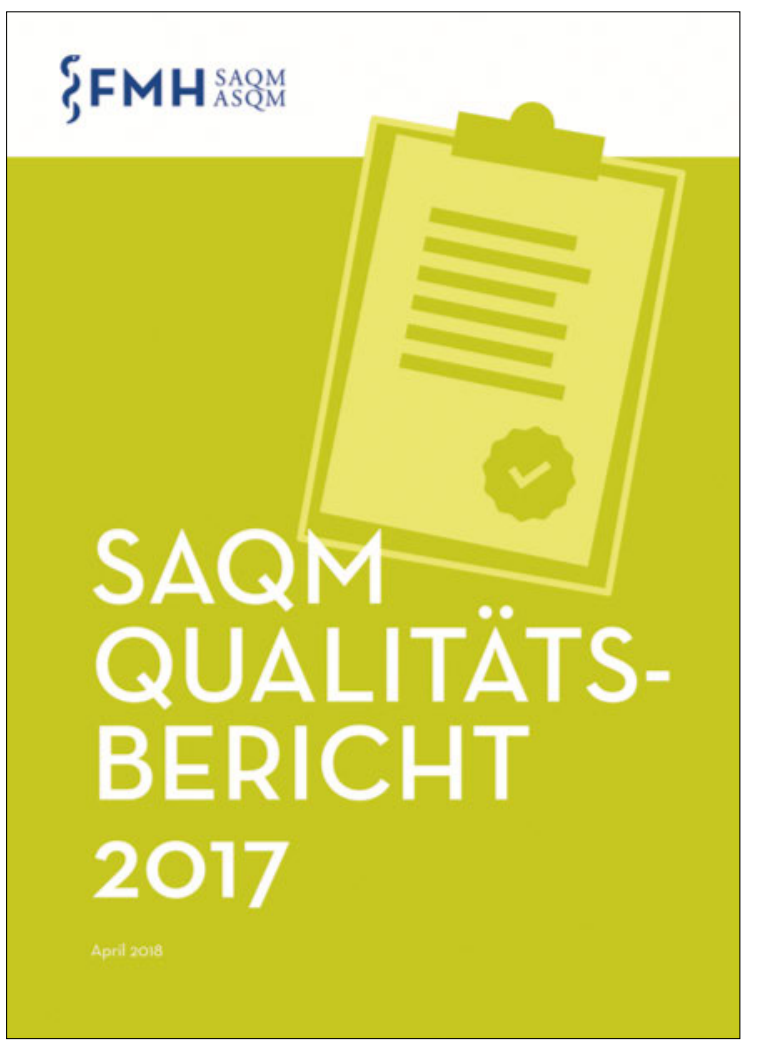


ziert. Den SAQM Qualitätsbericht in seiner vollen Länge finden Sie auf der SAQM-Website unter: www. saqm.ch $\rightarrow$ Qualitäts-Charta $\rightarrow$ Qualitätsberichte

\section{SAQM Qualitäts-Charta}

Die Qualitäts-Charta hält Grundsätze für Qualität in der Medizin schriftlich fest. Mit der freiwilligen Unterzeichnung der Charta bekennen sich Ärzteorganisationen zu Kooperation, Vernetzung und Transparenz in Qualitätsfragen in der Schweiz. Seit 2016 und noch immer ist die Unterzeichnung der Charta möglich. 2017 ist die 69. Ärzteorganisation der Charta beigetreten. Für sie alle gilt es nun, die Inhalte der QualitätsCharta mit einer Qualitätsstrategie und einem ersten Qualitätsbericht umzusetzen. 2017 wurden bereits sechs Qualitätsstrategien und drei Qualitätsberichte bei der SAQM eingereicht. Aktuell stehen wir bei acht Strategien und sechs Berichten (Stand: 3.4.2018). Weitere Informationen sind auf der Website aufgeführt unter: www.saqm.ch $\rightarrow$ Qualitäts-Charta

\section{SAQM-Qualitätspreis «Innovation Qualité»}

Der neue Qualitätspreis der SAQM zeichnet praxiserprobte Qualitätsprojekte des Schweizer Gesundheitswesens aus, vernetzt Vordenker der medizinischen Qualität aus verschiedensten Disziplinen und Berufen und präsentiert das vielfältige Qualitätswissen der Schweiz. Die Innovation Qualité prämiert Qualitätsprojekte in drei Kategorien:

- Themenschwerpunkt 2017: Patientenversorgung neu gedacht

- Patientensicherheit

- Ärzteorganisationen

2017 haben sich 37 Projektteams für die Innovation Qualité beworben. Am 17. April 2018 werden die Gewinner am SAQM-Symposium vorgestellt. Weiterführende Informationen finden Sie auf der SAQM-Website: www. innovationqualite.ch

\section{SAQM-Projekte (Auswahl, die vollzählige Auflistung finden Sie hier: www.saqm.ch $\rightarrow$ Qualitätsprojekte)}

1) Interprofessionelles Fortbildungsangebot Qualität

Die SAQM entwickelt ein interprofessionelles Fortbildungsangebot als CAS-Lehrgang zum Thema "Qualität in der Medizin für die patientennahe Arbeitspraxis». Das Ziel des Kurses ist, dass Gesundheitsfachleute einen souveränen Umgang mit der Thematik der Qualitätsarbeit in der Medizin be- herrschen: Die Kursteilnehmenden sind befähigt, eigenständig Projekte im Bereich Qualitätsentwicklung und Patientensicherheit zu entwickeln sowie umzusetzen.

2) Neue Online-Plattform "Guidelines Schweiz» Medizinische Guidelines sollen im Interesse der bestmöglichen Behandlung der Patientinnen und Patienten evidenzbasierte Entscheidungsgrundlagen zur Verfügung stellen. Der Guideline-Entwicklungsprozess hilft, sich auf einheitliche Behandlungsrichtlinien zu einigen. Die Online-Plattform «Guidelines Schweiz» dokumentiert Guidelines nach einer einheitlichen Struktur und bietet damit einen Überblick sowie Informationen über Guidelines. Zugang zur Plattform: https://www.guidelines.fmh.ch/index.cfm?l=1

\section{3) Pilotprojekt «Patient Centered Outcome Registry" (PCOR)}

Im Zentrum stehen dabei die Bedürfnisse des einzelnen Patienten im Kontext der Begleitumstände und Komorbiditäten. Gelingt es mittels der gemeinsam definierten Abklärungs- und Behandlungsschritte, das gemeinsam definierte Ziel zu erreichen? Dieses Instrument soll schliesslich BestPractice-Empfehlungen ermöglichen, welche auf Alltagserfahrungen basieren und so eine wertvolle Ergänzung zu den evidenzbasierten Guidelines bilden.

\section{4) Sektorenübergreifender Behandlungspfad Kolorektalkarzinom}

Im Rahmen des Pilotprojekts interprofessioneller sektorenübergreifender Behandlungspfad Kolorektalkarzinom (CRC) erarbeiteten und verabschiedeten in einem strukturierten Bottom-up-Prozess 20 in die Behandlung von Patientinnen und Patienten mit einem CRC involvierte Fachgesellschaften und Berufsgruppen die Grundlagen, damit alle Patientinnen und Patienten mit CRC, unabhängig von ihrem Wohnort in der Schweiz, eine qualitativ hochwertige, nach (inter-)nationalen evidenzbasierten Richtlinien bestmöglich koordinierte Versorgung erhalten. Das nun veröffentlichte schematische Pfadmodell ist bei allen CRC-Patientinnen und -Patienten anwendbar, weil es zusätzlich zu den Aspekten der primären Tumorerkrankung sowohl Komorbiditäten als auch Lebenserwartung berücksichtigt.

\section{5) Empfehlungen zum Aufbau und Betrieb} von gesundheitsbezogenen Registern Register mit verlässlichen Daten werden im Ge- 
sundheitswesen immer wichtiger und mit den wachsenden Datenmengen zahlreicher. Um zur Qualitätssicherung beizutragen, haben die Organisationen FMH, ANQ, H+, SAMW und unimedsuisse gemeinsam Empfehlungen für den Aufbau und Betrieb von gesundheitsbezogenen Registern herausgegeben. Diese enthalten Mindeststandards, unter anderem zum Datenschutz und zur Datenqualität. Die Empfehlungen bieten des Weiteren Grundlagen, um die Qualität laufender sowie künftiger Register zu prüfen. Weitere Informationen: www. saqm.ch $\rightarrow$ Publikationen $\rightarrow$ Empfehlungen Register

\section{SAQM-Publikationen}

Die SAQM publiziert regelmässig Grundlagenpapiere und Stellungnahmen, Projektberichte oder Medienmitteilungen.

2017 ist das Grundlagenpapier zum Thema "Choosing Wisely - für weniger unnötige Leistungen» erschienen. Auch wurden einige Artikel in der Schweizerischen Ärztezeitung zu spezifischen Themen im Bereich Qualität publiziert: FMH Ärztestatistik, Porträt über die SAQM, Online-Plattform für Qualitätsinitiativen und für Guidelines, Innovation Qualité sowie «Translationale Medizin" am SAQM-Symposium.

Zudem informiert Sie der SAQM-Newsletter ca. 5- bis 6-mal jährlich per E-Mail über Projekte, Arbeiten und neuste Publikationen der SAQM. Wir freuen uns über Ihr Interesse und Ihre Anmeldung via saqm[at]fmh.ch

\section{Position des Zentralvorstandes der FMH}

- Für Qualitätsarbeit und Qualitätsentwicklung soll auf bestehenden Organisationen aufgebaut werden. Damit wird Commitment geschaffen, werden Synergien genutzt und das konsolidierte Wissen der Organisationen eingebunden, welches höher zu werten ist als Expertenwissen.

- Die vielfältige Qualitätsarbeit der Ärzteschaft soll auch von Verwaltung und Politik anerkannt werden. Seitens Politik, Verwaltung und Kostenträger sind geeignete Finanzierungen und sinnvolle Rahmenbedingungen zu schaffen.

Korrespondenz:

FMH Verbindung der Schweizer Ärztinnen und Ärzte; Abteilung Daten, Demographie und Qualität Elfenstrasse 18

Postfach 300

CH-3000 Bern 15

Tel. 0313591111

roxane.kuebler[at]fmh.ch desselben oder Verweigerung sind Sanktionen sinnvoll. Als einziges oder primäres Instrument sind sie jedoch mit mehr Nebenwirkungen behaftet als mit Wirkungen.

\section{Ausblick 2018}

Auch in diesem Jahr macht sich die SAQM im Dienste der Ärzteschaft mit spannenden Projekten und Themenschwerpunkten für die Qualität in der Medizin stark:

- Empfehlungen zum Aufbau und Betrieb von gesundheitsbezogenen Registern

- Interprofessionelle Peer-Reviews für die Psychiatrie

- Grundlagenpapier zum Thema «Patient Reported Outcome Measures»

- Preisverleihung der Innovation Qualité am SAQMSymposium am 17. April 2018

- Begleitung der Umsetzung der Qualitäts-Charta

- Interprofessionelles Fortbildungsangebot Qualität

- Dialoggruppe Versorgungsforschung ISPM Bern, FMH, KKA und NewIndex

- Schaffung eines Labels für «Responsible Practice FMH»

Das grosse Spektrum der Projekte und Tätigkeiten der SAQM zeigt, dass sie in einem breiten Feld unseres Gesundheitswesens aktiv ist und als Ansprechpartner für Qualitätsthemen in der Medizin wahrgenommen wird. Im Zentrum der Arbeiten steht der Mehrwert, den kompetente Qualitätsarbeit für Patienten, Angehörige und Ärzte schafft.

\section{SAQM-Dienstleistungen für Qualität}

Die Dienstleistungen der SAQM stehen den Ärzte- und Partnerorganisationen sowie auch Einzelpersonen und Dritten zur Verfügung. In folgenden Bereichen kann eine Unterstützung durch die SAQM beantragt werden:

- Vernetzung und Koordination in medizinischen Qualitätsfragen

- Fachliche Unterstützung in Qualitätsfragen

- Eingabe neuer Themen

- Punktuelle finanzielle Unterstützung

Weitere Informationen erhalten Sie hierzu auf der SAQM-Website unter der Rubrik «Unterstützung» (www.saqm.ch $\rightarrow$ Dienstleistungen $\rightarrow$ Unterstützung).

\section{Bildnachweis}

FMH

Weiterführende Informationen: www.saqm.ch 